\title{
Estimating and determining the position of target in WSN's
}

\author{
K.Harshini ${ }^{1}$, Deepika Vodnala ${ }^{2}$ \\ M.Tech Student, SR Engineering College, Warangal, Telangana, India ${ }^{1}$ \\ Assistant Professor, SR Engineering College, Warangal, Telangana, India ${ }^{2}$
}

\begin{abstract}
In the proposed paper the major issue is the target tracking in wireless sensor networks and to obtain exact position of the target as to be measured so that we can expect improvement in the tracking resolution. so there is need of finding accurately sensor and targets trajectory path as to be studied with special care and captured at regular basis. We also studied correlation properties and sensitivity in mobile sensors from the system parameters and maintained good resolution for tracking of mobile sensors at different speeds. Our simulation results gave satisfactory system parameters like sensor density, sensor and target mobility compared static sensor network environment.
\end{abstract}

Keywords: Component, Mobile sensor networks, sensor range, resolution, Mobility Model

\section{INTRODUCTION}

Recently if we generally focus on target tacking by considering both moving target and mobile sensor network (MSN). Our main interests is in finding out the spatial resolution in order to localize the resolution of the target's trajectory and also find out optimization problem solution in Mobile sensor networks, by developing new algorithm that establishes an object tracking in wireless sensor networks. This algorithm will also help us to refine the sensor mobility model, the network model, and the communication model among sensors in order to enable effective detection and tracking. To capture all aspects of such tracking problems in real applications, one needs full descriptions of both the uncontrolled dynamics of the target as well as the controlled dynamics of the mobile sensors. In the applications of interest, sensors are often placed on mobile vehicles; hence, the control system describing a mobile sensor is in itself a complicated nonlinear system with a large state space. We derive spatial resolution that is between our assumed model and the target model travelling pathway which is also termed as distance that particular target is uncovered by any of the available mobile sensors. Also we need of obtaining of minimum no.of mobile sensors that could be deployed to properly establish accurate spatial resolution in present mobile wireless sensor networks. This shows the problem is very similar to collision problem in our classical kinetic theory of gas molecules that lets us to derive complete with dynamic relationship between our wireless mobile sensors and moving targets scenario.

\section{LITERATURE SURVEY}

Target tracking systems have certain real-time constraints for to even sudden transient changes for fast moving targets, on the other hard real time performance of these issues is major constraint in this type of applications that must be compatible with the present system properties like energy consumption and accuracy. Prediction and localisation of nodes by method of object monitoring Ant Optimization in Sensor Networks. Here once the object is determined, the mobile node moves to cover the particular object. If the Target cannot be defined then the set of new nodes are located and each node is assigned a position to minimize the total travelled distance. In this scheme estimation and prediction of nodes are done by Interval Theory and the Relocation of Nodes is done by using Ant Colony Optimization, find out the drawback of technology and the area in which sensor unable to track the user location. In the paper Protocol for tracking mobile targets using Wireless Sensor Networks employs an protocol distributed predictive tracking for tracking moving targets with use of sensor networks that uses an cluster based method for prediction and scalability based scheme that provides energy efficient solution. The proposed scheme is more robust for node as well as prediction a failure that yields temporary losses of targets and its recovery at the cost of extra energy usage.

Target tracking in distributed environment by developing protocol:

1) Clustering based approach for scalability and predication based tracking mechanism to provide distributive and energy efficient solution.

2) The power conversation is key factor which is taken in to consideration for execution of system.

\section{NETWORK AND MOBILITY MODEL}

We consider a mobile sensor network (MSN) to consist of $\mathrm{N}$ (A) mobile sensors initially placed inside a two dimensional geographical region shown in Figure1.

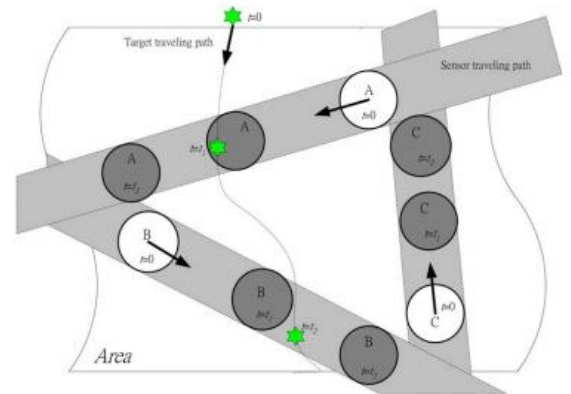

Fig. 1. Example of target tracking problem in a mobile sensor network. The difference between the intersections between sensor traveling path and target traveling path and the coverage for the target path. 
As shown in above figure region can be in any convex shapes under the proposed formulation. Here we are going to take a rectangular region which consists of four boundaries which helps us in considering it as a rectangle so that it will be easy in making any cautions which are required. As we are rectangle in assumption lets take the width of the rectangle with the area to be $\mathrm{W}$ and the length to be $|\mathrm{A}| / \mathrm{W}$, where $|\mathrm{A}|$ represents the area of the region. For the initial configuration (at time $\mathrm{t}=0$ ), we assume considered sensors are independently deployed at a random uniform distribution. Under this assumption, the sensor location can be modeled by a stationary twodimensional Poisson process. Denote the density of the Poisson process

as $n_{A}$. The number of sensors located in the region $A, N(A)$, follows a Poisson distribution of parameter $n_{A} \cdot|\mathrm{A}|$.

\section{A. Sensing and Mobility Model}

In this step every sensor all sensors have a sensing area which will be able to sense only the area or the region in which it is located and in the same way sensor will be able to detect the events which are occurring in and around the specific region. Here we are going to consider any object which is detected by the sensor and it is moving in the area as an target. A object is considered to be covered or detected when it is identified in the boundaries or in the limits of the region which is covered by the sensor. We assume the sensing region to be a disk of radius $\mathrm{R}$ centered at the sensor. In an MSN, depending on the mobile platform and application scenario, sensors can choose from a wide variety of mobility strategies, from passive movements to highly coordinated and complicated motion. The movement patterns are referred as the uncontrolled sensor mobility model simple robots may have a limited set of mobility patterns, whereas advanced robots can navigate in a more complicated itinerary. The movement patterns are referred as the controlled sensor mobility model. In this work, we consider the following uncontrolled sensor mobility model. We assume that sensors move independently of each other, without any coordination between them. The movement of a sensor is characterized by its speed and direction. A sensor travels to the boundary of area A at a chosen speed and direction. Once the boundary is reached, the sensor bound back, by choosing another angular direction and continues the process, so we termed it as random direction mobility model. We assume the velocity of a target is a constant $v_{i}$, which is defined as a path (line segment) crossing from one boundary to another.

\section{B. Tracking Measurement}

Here spatial resolution in MSNs as the average deviation between actual assumed as well as actual target travel paths, so therefore to find deviation between the assumed and the actual paths can be illustrated below depicts the distance so target is not covered by any of the sensors. From figure 2, the target is covered by sensors under the time periods ( $\mathrm{t} 1$ to $\mathrm{t} 2$ ) and ( $\mathrm{t} 3$ to $\mathrm{t} 4$ ), while it cannot be localized by any sensors before $t 1$, after $t 4$ and between $t 2$ to $\mathrm{t} 3$.

\section{TARGET TRACKING IN A MOBILE SENSOR NETWORK}

Here we can treat a mobile sensor as a gas molecule, and a target as an electron.

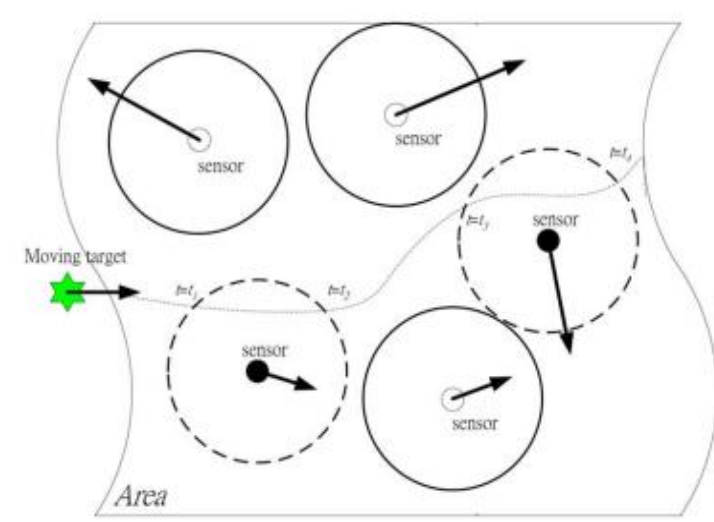

Fig. 2. The spatial resolution of a mobile sensor network.

\section{A. Spatial resolution}

Here we took the average deviation between the estimated and the actual target travel paths, which is the average travel distance of a target between successive coverage by mobile sensors. Initially we assume sensors are stationary and, then extend our formulation to consider sensor mobility. Let sensing range is $R$, when $t=0$, a cross section of coverage can be modeled by using a circle with the diameter $2 \mathrm{R}$. After a period of time $\Delta \mathrm{t}$, the circle swept out an area (shown in Figure 3 ) and the amount of sensor coverage can be estimated from the density of mobile sensors $(\mathrm{nA}=\mathrm{N}(\mathrm{A}) /|\mathrm{A}|)$ inside the area.

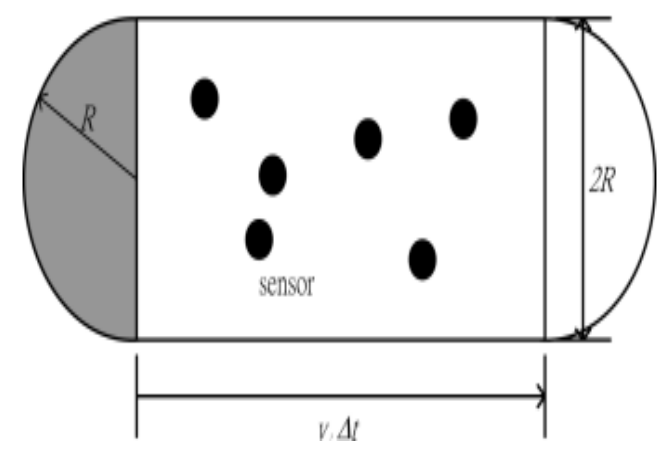

Fig. 3. An effective coverage region with sensing range $\mathrm{R}$ at time $\mathrm{t}=\tau$.

\section{B. Relative speed under the random direction mobility model}

The speed of a moving target relative to mobile sensors varies only with the angle between their respective directions of movement, which is shown in Figure 4a.

Since the mobile sensors move randomly in all possible directions (due to the random directional mobility model), a fraction $d \theta / 2 \pi$ of them move in directions that are within angle $\mathrm{d} \theta$ of the target vi direction. 


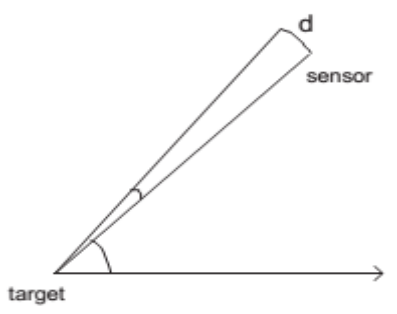

(a)

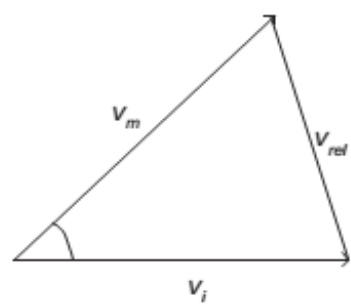

(b)

Fig. 4. (a)The speed of the moving target relative to one of mobile sensors varies only with the angle between their respective directions of motion;

(b)The relative speed $v_{\text {rel }}$

\section{B. Distribution of spatial resolution}

We use the average deviation instead of the maximum deviation for the definition of spatial resolution in MSNs, we study the probability distribution function of the uncovered distance. We consider a group of targets to be initially outside the region. Let the number originally in the group at time $\mathrm{t}=0$ be $\mathrm{N} 0$, and at time $\mathrm{t}, \mathrm{N}$ of them going without coverage by any mobile sensors.

Let 1 be the uncovered distance for the length $v_{i} t$, where a target has been covered at time t. The number of targets that are covered between and $\mathrm{t}+\mathrm{dt}$ and terminate a path whose length lies between 1 and $1+\mathrm{dl}$ is

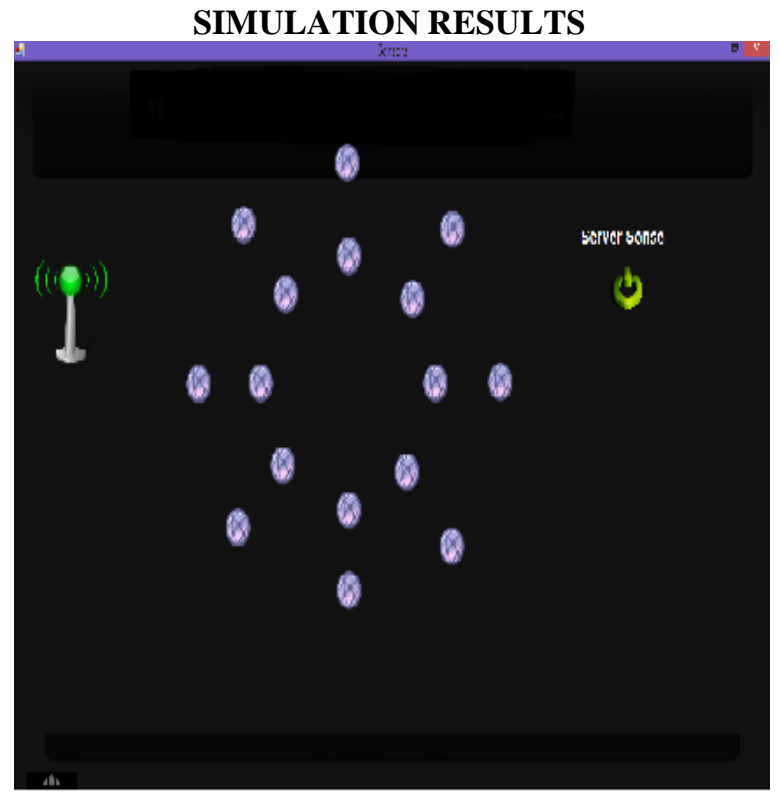

Fig. 5. This figure demonstrates the placement of sensors which are are in ready mode to detect when ever there is any movenent in the objects.

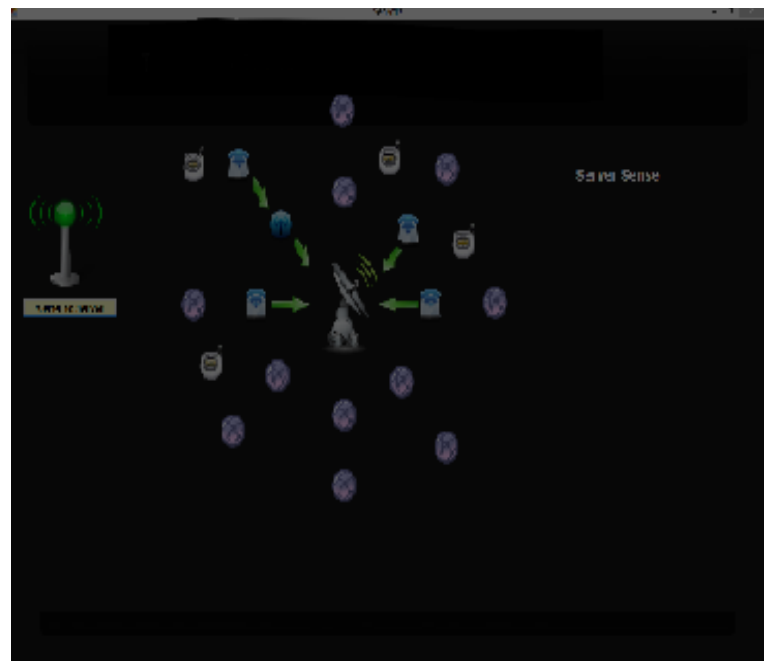

Fig. 6. This figure demonstrates the the difference between the intersections between sensor traveling path and target traveling path and the coverage for the target path.

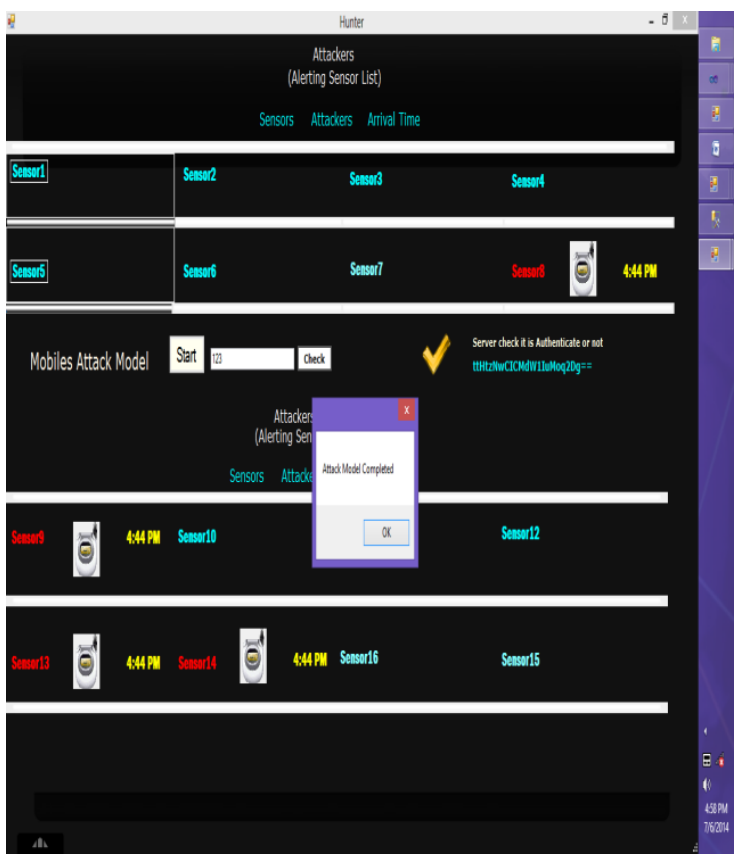

Fig. 7. Provideing the information about the venerabel sensors when the information is sent.

\section{CONCLUSION}

In this proposed model, we have concentrated the major target tracking problem in mobile wireless sensor networks. Generally, we analyzed performance metrics like: spatial resolution and we also investigate the resolution of moving targets. We also studied are several issues for finding research on this problem: (1) to obtain the detection error of mobile sensors under different varying wireless sensor speeds, which in turn makes up an optimization problem for target tracking; (2) to further refine the sensor mobility model, the network model, and the communication model among different sensors in establishes effective detection and tracking. 


\section{ACKNOWLEDGEMENT}

I am extremely grateful to those who have helped, contributed and supported us during the project. Our deepest thanks to our advisor Deepika Vodnala for her continuous encouragement, for explaining many scenarios where the project can be used and suggestions throughout the course of this work. It was our pleasure to work under her guidance.

\section{REFERENCES}

[1] D. Aberdeen, "A (Revised) Survey of Approximate Methods for Solving Partially Observable Markov Decision Processes," National ICT Australia, Canberra, Australia, 2003.

[2] N. Shrivastava, R. Mudumbai, U. Madhow, and S. Suri. Target tracking with binary proximity sensors: Fundamental limits, minimal descriptions, and algorithms. In Proc. of SenSys. ACM, October 2006.

[3] J. Aslam, Z. Butler, F. Constantin, V. Crespi, G. Cybenko, and D. Rus.Tracking a moving object with a binary sensor network. In Proc. Of Sen Sys. ACM, November 2003.

[4] W. Kim, K. Mechitov, J.-Y. Choi, and S. Ham. On target tracking with binary proximity sensors. In Proc. of IPSN. IEEE, April 2005.

[5] J. Singh, U. Madhow, R. Kumar, S. Suri, and R. Cagley. Tracking multiple targets using binary proximity sensors. In Proc. of IPSN.ACM, April 2007

[6] H. Zhang and J. C. Hou. Maintaining sensing coverage and connectivity in large sensor networks. Ad Hoc and Sensor Wireless Networks, 1(1-2):89-124, March 2005.

[7] Niranchana, S. ; Dinesh, E. ieee paper"Object monitoring by prediction and localisation of nodes by using Ant Colony Optimization in Sensor Networks" Advanced Computing (ICoAC), 2012 Fourth International Conference in Chennai 13-15 Dec. 2012.

\section{BIOGRAPHIES}

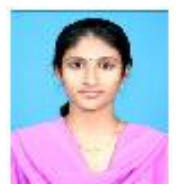

K.Harshini received the btech degree in computer science and enginnering from balaji institute of technology and sciences,laknepally,narsampet Currently Pursuing M.tech in Computer Science and Engineering at SR Engineering College,Warangal, India. Her research interests include in networks, Mobile Computing.

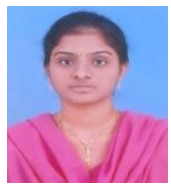

Deepika Vodnala received Bachelor of Technology Degree in Information Technology from Kamala Institute of Technology \& Sciences (affiliated to JNTU Hyderabad), Karimnagar, Telangana, India, and Master of Technology Degree in the stream of Software Engineering from Ramappa Engineering College (affiliated to JNTU Hyderabad), Warangal, Telangana, India. She is pursuing Ph.D. in the area of Mobile ad-hoc networks in GITAM University, Hyderabad, Telangana, India. She has 4 years of teaching experience. Presently she is working as Assistant Professor, Department of Computer Science and Engineering in SR Engineering College (Autonomous, affiliated to JNTU Hyderabad), Warangal, Telangana, India. She has published Four International Journal Papers and Four International Conference Papers. Her research interests are Mobile Adhoc Networks and Wireless sensor Networks. She is a Life Member of Indian Society for Technical Education (ISTE). 